\title{
Renormalization group irreversible functions in more than two dimensions
}

\author{
José Gaite* \\ Centro de Astrobiología, Instituto Nacional de Técnica Aeroespacial, Ctra. de Torrejón a Ajalvir, \\ 28850 Torrejón de Ardoz, Madrid, Spain
}

(Received 15 May 2000; published 29 November 2000)

\begin{abstract}
There are two general irreversibility theorems for the renormalization group in more than two dimensions: the first one is of entropic nature, while the second one, by Forte and Latorre, relies on the properties of the stress-tensor trace, and has been recently questioned by Osborn and Shore. We start by establishing under what assumptions this second theorem can still be valid. Then it is compared with the entropic theorem and shown to be essentially equivalent. However, since the irreversible function of the (corrected) Forte-Latorre theorem is nonuniversal (whereas the relative entropy of the other theorem is universal), it needs the additional step of renormalization. On the other hand, the irreversibility theorem is only guaranteed to be unambiguous if the integral of the stress-tensor trace correlator is finite, which happens for free theories only in dimensions smaller than four.

PACS number(s): 11.10.Gh, 04.62.+v, 11.10.Kk
\end{abstract}

The search for a function representing the irreversible nature of the coarse-graining transformations of Wilson's renormalization group $(\mathrm{RG})$ has a long history. After the success of Zamolodchikov's $c$-function in two dimensions (2D), it was shown that a straightforward generalization to a higher dimension was not possible but, at the same time, it was observed that a related function, the integral of the stress-tensor trace on a constant curvature space, could play a similar role [1]. In an interesting article [2], Forte and Latorre formulated an irreversibility theorem in terms of this quantity. However, an exhaustive analysis of this theorem carried out by Osborn and Shore [3] shows that there were missing terms in that theorem that actually spoil the irreversible character of that function.

In a separate development, we have introduced in field theory the relative entropy, a quantity borrowed from probability theory, which turns out to be the Legendre transform of $W(\lambda)-W(0)$ with respect to $\lambda[4]$ :

$$
\begin{aligned}
S_{\text {rel }}(\lambda) & =W(\lambda)-W(0)-\lambda \frac{d W}{d \lambda} \\
& =W-W_{0}-\lambda\left\langle f_{\lambda}\right\rangle,
\end{aligned}
$$

where $f_{\lambda}$ is a composite field integrated over the whole space, $f_{\lambda}=\int d^{D} x \Phi(x)$. As a straightforward consequence of its definition, the relative entropy satisfies a monotonicity theorem

$$
\begin{aligned}
\frac{d S_{\text {rel }}}{d \lambda} & =\frac{d W}{d \lambda}-\frac{d}{d \lambda}\left(\lambda \frac{d W}{d \lambda}\right)=-\lambda \frac{d^{2} W}{d \lambda^{2}} \\
& =-\lambda \frac{d}{d \lambda}\left\langle f_{\lambda}\right\rangle=\lambda\left\langle\left(f_{\lambda}-\left\langle f_{\lambda}\right\rangle\right)^{2}\right\rangle \geqslant 0,
\end{aligned}
$$

\footnotetext{
*Also at Instituto de Matemáticas y Física Fundamental, CSIC, Serrano 123, 28006 Madrid, Spain.
}

which can be interpreted as showing the irreversibility of the $\mathrm{RG}[4,5]$. As we remarked in previous papers, the relative entropy is not the only monotonic quantity with the RG. For example, from the same equation that shows its monotonicity (2), one can realize that the function $\left\langle f_{\lambda}\right\rangle=d W / d \lambda$ is monotonic as well.

Let us consider the integral $\int d^{D} x\langle\Theta(x)\rangle$, where $\Theta$ is the stress tensor trace. In a homogeneous space, the expectation value $\langle\Theta\rangle$ is independent of the position and only depends on the coupling constants; hence, the integration is trivial, its only effect being to produce an overall factor. We further consider a field theory with simple scaling behavior, namely, with only one coupling constant such that $\lambda \propto m^{y}$, where $m$ is the physical mass of the fundamental particle or some other mass scale. This behavior is very common in critical phenomena. Since $\Theta$ gives the response to a change of the scale $m$,

$$
\langle\Theta\rangle \equiv m \frac{d W}{d m}=y \lambda \frac{d W}{d \lambda}=y \lambda\langle\Phi\rangle
$$

where $W$ is now a specific quantity (per unit volume). In other words, the expectation value of $\Theta$ is proportional to the monotonic function $\left\langle f_{\lambda}\right\rangle$. By substituting for it in the monotonicity Eq. (2), we can write this equation in the suggestive form

$$
-m \frac{d}{d m}\left(m^{-y}\langle\Theta\rangle\right)=m^{-y} \int d^{D} x\langle\Theta(x) \Theta(0)\rangle_{c},
$$

where the subscript $c$ means that one is to take the connected correlation function. The integral of this correlation function may be divergent. If $m \neq 0$ it converges for $x \rightarrow \infty$. On the other hand, the behavior of the two-point function for $x \rightarrow 0$ is the same as in the massless $(\lambda=0)$ theory, thus given by the dimension of $\Phi, d_{\Phi}$. Therefore, the integral is UV convergent if $2 d_{\Phi}<D$, that is, if $y=D-d_{\Phi}>D / 2$. One can also derive an equation for $\langle\Theta\rangle$ : 


$$
-m \frac{d}{d m}\langle\Theta\rangle=\int d^{D} x\langle\Theta(x) \Theta(0)\rangle_{c}-y\langle\Theta\rangle .
$$

In Euclidean space, the form of the quantities defined above is given by scaling (e.g., $S_{\text {rel }} \propto m^{D}$ ) and has little physical content. In a curved homogeneous space one can form the dimensionless variable $u=R m$, where $R$ is the curvature radius, and dimensionless quantities are non-trivial functions of it. In particular, we have the dimensionless function of Refs. [1-3], $c(u)=R^{D}\langle\Theta\rangle$. Introducing a constant curvature space has an additional utility: Eq. (5) can also be obtained starting from the scale Ward identities satisfied by the energy momentum tensor as $R$ varies [2,3]. Let us remark that the derivation in Ref. [2] yields a slightly different equation. It has been polished in Ref. [3], to obtain an equation similar to but more general than Eq. (5):

$$
\begin{aligned}
-R \frac{d}{d R}\left(R^{D}\langle\Theta\rangle\right)= & R^{D} \int d^{D} x\langle\Theta(x) \Theta(0)\rangle_{c} \\
& -R^{D} \beta^{i}\left(\partial_{i} \mathcal{A}+\partial_{i} \beta^{j}\left\langle\Phi_{j}\right\rangle\right) .
\end{aligned}
$$

This equation takes into account the possibility of several couplings and the existence of the trace anomaly $\mathcal{A}$, such that $T_{a}^{a}=\Theta+\mathcal{A}$, where $\Theta=\beta^{i} \Phi_{i}$. We can convert Eq. (6) into Eq. (5) by (i) assuming simple scaling behavior, that is, with only one coupling such that $\beta=y \lambda$, the anomaly $\mathcal{A}$ being independent of it, and by (ii) replacing the derivative with respect to $R$ with a derivative with respect to $m$.

Therefore, even though in the general case no monotonicity theorem seems to follow from Eq. (6) [3], it does in our case, namely, the one expressed by Eq. (4). However, the monotonic quantity (with respect to $m$ or $R$, indistinctly) is not just $c(u)=R^{D}\langle\Theta\rangle$, as proposed in Refs. [1-3], but rather $\tilde{c}(u)=u^{-y} c(u)$. They only coincide if $y=0$, that is, when the coupling constant is dimensionless. Generally, the functions $c$ or $\tilde{c}$, involving the composite field $\Phi$, contain (normal order) UV divergences. We can introduce a UV regulator but, given that it can only be removed by introducing another scale (renormalization point), those functions are not universal.

To define a finite monotonic function from the stresstensor trace, one has, therefore, to perform a subtraction. Let us define the function

$$
f(u)=-V_{D-1} m^{-y} R^{D-y}\langle\Theta\rangle,
$$

where $V_{D-1}=2 \pi^{D / 2} / \Gamma(D / 2)$ is the volume of the unit ( $D$ $-1)$-dimensional sphere. This function is essentially $\tilde{c}(u)$, except for a conventional sign (to make it increasing rather than decreasing) and a normalization factor. It is UV divergent but, assuming the convergence of the integral in Eq. (4), one subtraction suffices to render it finite. The point is that when integrating $d f / d m$ according to Eq. (4), one has an integration constant which can be infinite. Therefore, we can define a renormalized value as [6]

$$
f_{\text {ren }}(m R):=\lim _{\Lambda \rightarrow \infty}\left[f_{\Lambda}(m R)-f_{\Lambda}\left(m_{0} R\right)\right]
$$

$\Lambda$ and $m_{0}$ being the UV cutoff and the subtraction point, respectively. In particular, one can set $m_{0}=0$. Alternatively, one can use minimal subtraction, by which one only subtracts the divergent part of $f$, which is independent of $m$ [7]. In any renormalization scheme we use the freedom afforded by the integration constant of Eq. (4), for example, to make $f(0)=0$, which is equivalent to taking $m_{0}=0$.

In contrast, the relative entropy is universal [under the assumption of convergence of the integral in Eq. (4)] because the UV divergences of $W$ cancel in the definition of $S_{\text {rel }}$, Eq. (1). One can define a dimensionless growing entropy $S$, proportional to $S_{\text {rel }}$. In terms of the function $f$,

$$
S(u)=y \int_{0}^{u} d v v^{y-1} f(v)-u^{y} f(u) .
$$

The renormalization constant of $f$ cancels in this formula.

To illustrate the general theory, we will study a free massive scalar field $\phi$, with coupling constant $\mathrm{m}^{2}$, in $D$-dimensional hyperbolic space $H^{D}$, where $D=2,3,4$. Naturally, a free massive scalar field theory is the simplest example of simple scaling one can take. The field expectation value $\left\langle\phi^{2}\right\rangle$ is then the Gaussian model energy $U\left(\mathrm{~m}^{2}\right)$ $[5,6]$, while $S_{\text {rel }}$ is a real thermodynamic entropy. Some expressions for the quantities in $D=2$ have been calculated in Ref. [6], in terms of the variable $r=(R m)^{2}$. More extensive calculations of $\left\langle\phi^{2}\right\rangle$ are given by Osborn and Shore [7]. For $H^{2}$,

$$
f_{\text {ren }}(r)=\psi(\sqrt{r+1 / 4}+1 / 2)+\gamma,
$$

where $\psi$ is the digamma function and $\gamma$ is the Euler constant. $f$ increases with $r$, on account of the properties of $\psi$.

For $D=3$ we could use the results of Osborn and Shore [8], but it is easier to use the heat-kernel method [6] since the $D=3$ heat kernel is extremely simple [9]:

$$
K(0 ; t)=\frac{e^{-t}}{(4 \pi t)^{3 / 2}} \text {. }
$$

Hence,

$$
\begin{aligned}
f_{\text {ren }}(r) & =-4 \pi \int_{0}^{\infty} \frac{d t}{(4 \pi t)^{3 / 2}}\left[e^{-(r+1) t}-e^{-t}\right] \\
& =\sqrt{r+1}-1 .
\end{aligned}
$$

This function is obviously increasing.

In $D=4$ (the case used as an example in Ref. [2]), $f$ has an expression similar to the one for $D=2[10,7]$. However, it is a particularly interesting case because the integral in Eq. (4) is now divergent, so $f_{\Lambda}^{\prime}(r)$ must be subtracted too. Consequently, two subtractions on $f$ are needed now, that is, 


$$
f_{\text {ren }}(r)=\lim _{\Lambda \rightarrow \infty}\left[f_{\Lambda}(r)-f_{\Lambda}\left(r_{0}\right)-\left(r-r_{0}\right) f_{\Lambda}^{\prime}\left(r_{0}\right)\right] .
$$

Subtraction at $m_{0}=0$ yields

$$
\begin{aligned}
f_{\text {ren }}(r)= & -\frac{1}{4}[(r+2) \psi(\sqrt{r+9 / 4}+1 / 2) \\
& \left.-2(1-\gamma)-\left(3-9 \gamma+\pi^{2}\right) \frac{r}{9}\right],
\end{aligned}
$$

which decreases for $r>0$. The reason is the following: One can compute $f_{\Lambda}^{\prime}(r)$, and it is indeed positive for sufficiently large $\Lambda$, since it diverges as $\ln \left(\Lambda^{2} / r\right)$. However, the subtraction removes precisely this dominant growing term. Given that the function $f^{\prime \prime}(r)$ is negative (besides finite), $f_{\text {ren }}^{\prime}(r)$ $<f_{\text {ren }}^{\prime}\left(r_{0}\right)$ if $r>r_{0}$. This could induce one to try to make the subtraction at the highest $r_{0}$ possible. This might be the idea behind the procedure proposed in Ref. [2], where it is demanded that $\lim _{r \rightarrow \infty} f_{\text {ren }}(r)=0$. However, this prescription implies subtracting from $f$ a function that is not a first degree polynomial in $r$, unlike in standard renormalization prescriptions, as exposed here (see also [7]).

Similar but more complicated expressions are obtained for the positive curvature case, the $D$-dimensional sphere $S^{D}$. In this case, one must also consider that, for $r=0$, the zero mode must be removed from the discrete spectrum, as done for $D=2$ in Ref. [6]. This subtraction, however, does not spoil positivity of the second term in Eq. (2).

Let us clarify the role of the trace anomaly, $\mathcal{A}$. It is well known that renormalization of the free action on a curved even-dimensional spacetime demands the presence of a term proportional to the curvature. It absorbs a logarithmic divergence that appears in addition to the logarithmically divergent term proportional to $m^{2}$ that is present on the plane [11]. Thus, the logarithmic derivative of $W$, with respect to the scale $R$, has two components: the stress-tensor trace on the plane $\Theta$, plus an additional part, independent of $m$ and proportional to $R^{-D}$ : the trace or conformal anomaly. The alert reader may have noticed that the original form of the $R$-monotonicity theorem (6) in Ref. [3] has $R^{D}\left\langle T_{a}^{a}\right\rangle$ in place of $R^{D}\langle\Theta\rangle$, but it does not matter because the difference is a constant. Nevertheless, adding this constant would have been a convenient normalization for the critical value of the monotonic quantity, had it been precisely $c(u)=R^{D}\left\langle T_{a}^{a}\right\rangle$, as proposed in Refs. [1,2], because it would make it proportional to the conformal central charge. However, since the correct monotonic quantity is, rather, $\tilde{c}(u)=u^{-y} R^{D}\langle\Theta\rangle$, adding the conformal anomaly would result in a divergence at the critical point.

Let us say a few words about the flat space limit $R \rightarrow \infty$. To take this limit, the function $f$ is no longer appropriate, and one must instead consider a local quantity, such as $R^{y-D} f(m R)=-V_{D-1} m^{-y}\langle\Theta\rangle$. Thus, for the massive free field theory in $D=3, \lim _{R \rightarrow \infty} R^{y-D} f(m R)=m$. In contrast, for $D=2, R^{0} f(m R)=f(m R)$ diverges logarithmically as $R$ $\rightarrow \infty$, as deduced from the corresponding asymptotic expansion [6]. This is because $R$ plays the role of an IR cutoff, and $f(0)$ is both IR divergent and UV divergent on the plane. The solution is to subtract at $r_{0} \neq 0$ before taking the limit, which will depend on $m_{0}$ and, therefore, one cannot construct a universal quantity. The same problem exists in $D$ $=4$, even though in this case, one should not give particular value to the point $m_{0}=0$ (as remarked above). Let us note, in passing, that the leading terms of the asymptotic expansion of $f(u)$ yield the flat space limit and, furthermore, for even dimensions, the sub-leading term yields the conformal anomaly [6].

In conclusion, the monotonicity theorems for the relative entropy or the stress-tensor trace are contained in Eq. (2). In field theory, $\left\langle\left(f_{\lambda}-\left\langle f_{\lambda}\right\rangle\right)^{2}\right\rangle$ is proportional to the integral of the stress-tensor trace correlation, which only converges if $y$, the dimension of the coupling constant $\lambda$, satisfies $y>D / 2$. Therefore, only under this condition is the irreversibility theorem unambiguous. However, even in this case, the function $f$ associated to the stress-tensor trace is ambiguous (nonuniversal), being defined only up to a constant, whereas the relative entropy is unambiguous (universal). After renormalization, the ambiguity of $f$ is realized as a dependence on $m_{0}$, which is the renormalization point in the simple scheme used here. Setting $m_{0}=0$ achieves a kind of universality, in the sense that no additional scale remains. But it may not be realizable, as occurs for free field theory on the plane. The case $y>D / 2$ covers many of the critical models of statistical mechanics, e.g., the 3D Ising model universality class, with $y=1.59$ [12]. When $y \leqslant D / 2$ (in particular, for bosonic freefield theory in $D=4$ ), the integral in the right-hand side of Eq. (4) is UV divergent and must be renormalized, in general spoiling its positivity, so the irreversibility theorem is itself ambiguous and may only hold in a particular renormalization scheme. Accordingly, $f$ needs to be subtracted twice. Of course, the problem of the divergence of that integral also affects the relative entropy, which becomes nonuniversal, requiring one additional subtraction further to those implied in its definition Eq. (1). Hence, it is doubtful whether one can assign an unambiguous meaning to RG irreversibility for $y$ $\leqslant D / 2$.

Since irreversibility in terms of the stress-tensor trace or the relative entropy are essentially equivalent, one may wonder which formulation is better. From a physical point of view, the theorem for the relative entropy has more content, being related to important notions in information theory $[4,13]$. From a mathematical point of view, $\langle\Theta\rangle$ is simpler to calculate and, in fact, to calculate $S_{\text {rel }}$, one must calculate it before [as in Eq. (9)].

I thank Hugh Osborn for both a conversation and for patiently explaining to me tricky points on some calculations in Ref. [3]. 
[1] J. Cardy, Phys. Lett. B 215, 749 (1988).

[2] S. Forte and J.I. Latorre, Nucl. Phys. B535, 709 (1998).

[3] H. Osborn and G.M. Shore, Nucl. Phys. B571, 287 (2000).

[4] J. Gaite and D. O'Connor, Phys. Rev. D 54, 5163 (1996).

[5] J. Gaite, Phys. Rev. Lett. 81, 3587 (1998); Phys. Rev. D 61, 045006 (2000).

[6] J. Gaite, Phys. Rev. D 61, 084001 (2000).

[7] In Ref. [3], Appendix D, $\langle\Theta\rangle=m^{2}\left\langle\phi^{2}\right\rangle$ is computed for free theories in various dimensions with dimensional regularization and minimal subtraction.

[8] In Ref. [3], the authors consider the scalar conformally coupled field theory, with curvature coupling $\xi=(D$ $-2) /[4(D-1)]$, whereas here we set $\xi=0$. Consequently, for $D=3,4$, some inessential numbers differ.

[9] R. Camporesi, Phys. Rep. 196, 1 (1990).

[10] R. Camporesi and A. Higuchi, Phys. Rev. D 45, 3591 (1992).

[11] N. D. Birrell and P. C. W. Davies, Quantum Fields in Curved Space (Cambridge University Press, Cambridge, England, 1982).

[12] J. Zinn-Justin, Quantum Field Theory and Critical Phenomena (Oxford University Press, Oxford, England, 1993).

[13] J. Preskill, J. Mod. Opt. 47, 127 (2000), Sect. 3.4. 